\title{
Serial Sectioning of Deformed Microcrystals using a Dual-Beam FIB-SEM Microscope
}

\author{
Michael D. Uchic* \& Paul A. Shade** \\ *Air Force Research Laboratory, Materials \& Manufacturing Directorate, AFRL/RXLMD, \\ Wright Patterson AFB, OH 45433 \\ ** Universal Technology Corporation, 1270 N Fairfield Road, Dayton, OH 45432
}

This paper describes the application of a Dual Beam Focused Ion Beam-Scanning Electron Microscope (DB FIB-SEM) to characterize the precipitate microstructures of deformed nickel superalloy microcrystals (i.e., miniature single-crystal mechanical test samples) in three dimensions. The microcrystals were fabricated via Focused Ion Beam milling of a bulk sample, and subsequently compressed using a modified nanoindenter [1]. The mechanical properties of these small samples are amenable to modeling via discrete dislocation simulations [2], which track the motion of all of the dislocations in such a volume. Precipitation hardening is an important strengthening mechanism for the alloy studied here, a nickel base superalloy (Rene N5). Thus, this study sought to provide realistic instantiations of these microstructures for potential use in discrete dislocation simulations. The microcrystals that were examined in this study were lightly deformed, as shown in Figure 1, in order to determine the mechanical properties of these samples prior to the destructive serial sectioning process.

Dual Beam microscopes have been previously shown to be capable of performing in-situ, highfidelity serial sectioning experiments without significant user supervision. In this study, we have employed both a FEI Strata DB 235 and a FEI Nova Nanolab microscope to perform the serial sectioning experiments. The serial sectioning thickness ranged from 25 to 50 nanometers, and the interrogated cylindrical sample volumes were approximately $5 \mu \mathrm{m}$ in diameter by $12 \mu \mathrm{m}$ in length. Prior to performing the serial sectioning experiment, the orientation of the primary slip system was identified via tilting experiments, and the serial sectioning plane was selected to be parallel to this plane. Next, the outer sample surface was capped with Pt using the GIS deposition, and three fiducial marks were milled into the area surrounding the microcrystals. These fiducal marks (in conjunction with custom control scripts that were developed to automate the collection of serial sectioning data) were used to ensure that sample position was consistent prior to cutting or imaging. The experiments collecting ion-induced secondary electron images, as this imaging condition provided the strongest contrast difference between the precipitate and matrix phase, albeit a lower ion beam current $(100 \mathrm{pA})$ was used to collect the image data than was used for serial milling (300 pA). In the associated presentation, we will discuss the collection and post-processing of the serial sectioning data, segmentation routines for semiautomated identification of the precipitate microstructure, and 3D reconstruction results.

[1] Uchic MD, Dimiduk DM. 2005 Mater. Sci. Eng. A 400-401:268-278

[2] Rao SI, Dimiduk DM, Parthasarathy, TA, Uchic MD, Tang M, Woodward C. 2008. Acta Mater. 56:3245-3259 


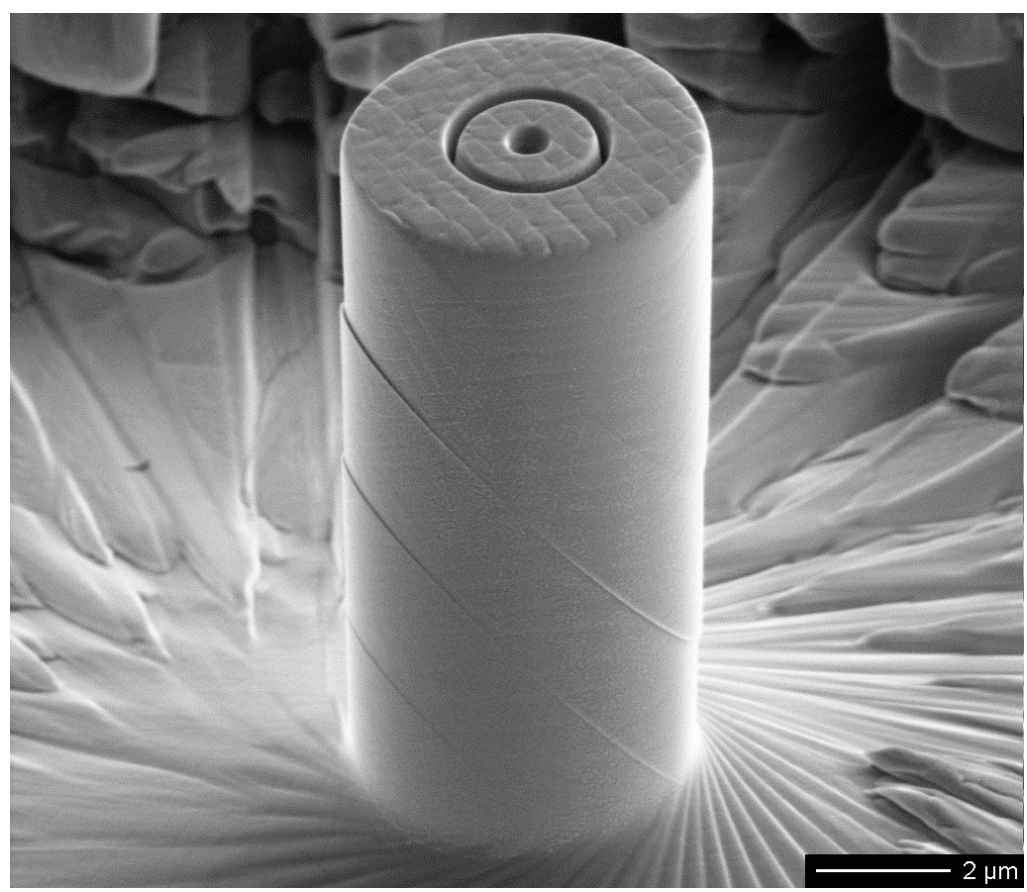

Figure 1. Scanning Electron Microscope image of a $5 \mu \mathrm{m}$ diameter deformed microcrystal of Rene N5. The sample has undergone $\sim 2.6 \%$ plastic strain, and one can observe 4 slip traces that have formed during the compression experiment.

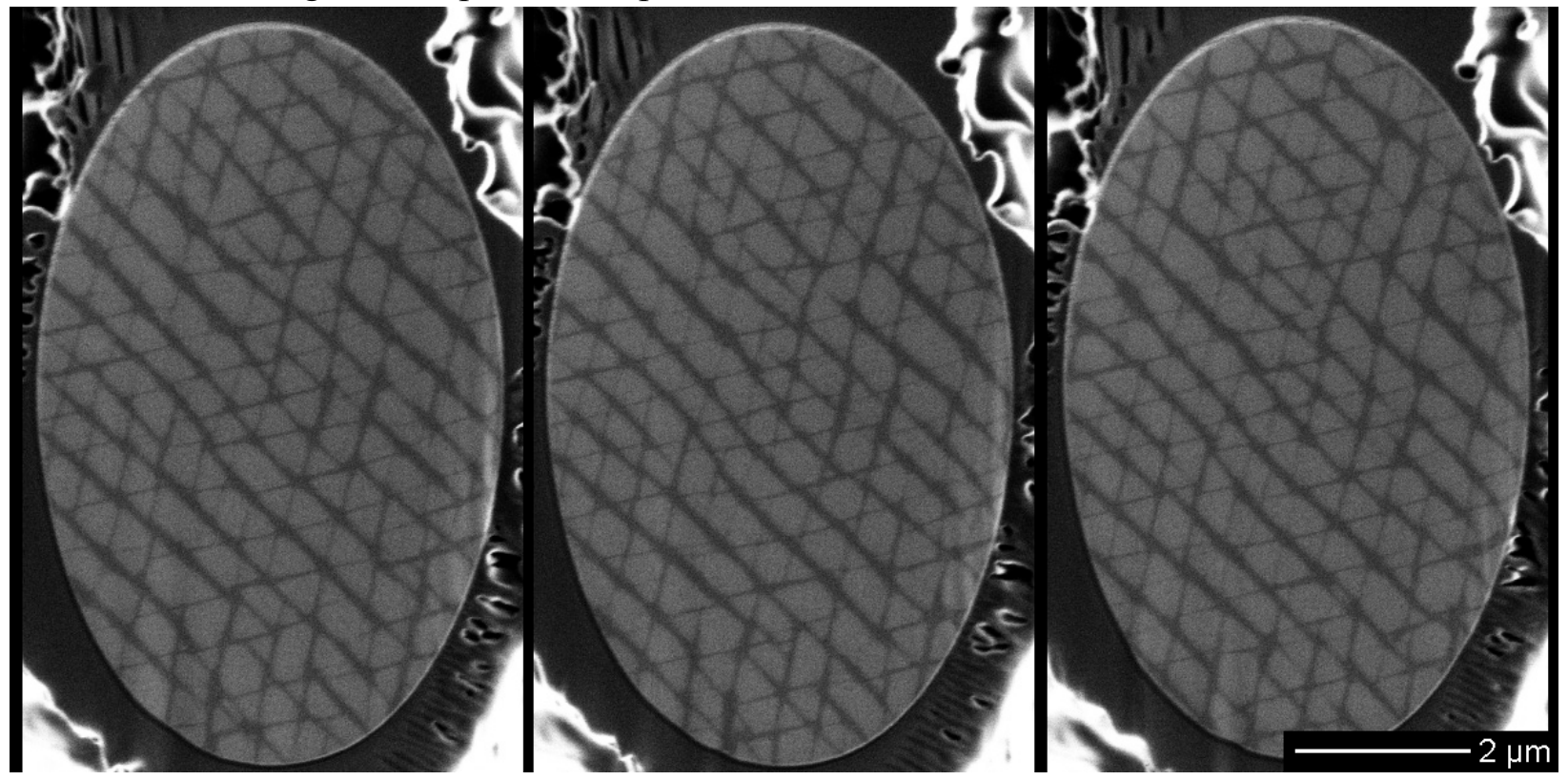

Figure 2. Three ion-induced secondary electron images from the serial sectioning experiment for the microcrystal shown in Figure 1. The lighter grey regions correspond to $\mathrm{Ni}_{3} \mathrm{Al}\left(\gamma^{\prime}\right)$ precipitates that reside within a Ni solid solution matrix $(\gamma)$, which is a darker grey color. The orientation of the serial-sectioning plane corresponds to the $<111>$ plane associated with the primary slip system. The change in depth for the images shown here is $100 \mathrm{~nm}$. 